\title{
GRADIS - Multiagent Environment Supporting Distributed Graph Transformations
}

\author{
Leszek Kotulski \\ Department of Automatics, AGH University of Science and Technology \\ Al. Mickiewicza 30, 30059 Krakow, Poland \\ kotuslki@agh.edu.pl
}

\begin{abstract}
Graph transformations are a powerful notation formally describing different aspects of modeled systems. Multiagent systems introduce distribution, parallelism and autonomous decision properties. In the paper a basic properties of the GRADIS agent's framework, joining of both approaches, are discussed. This framework supports splitting the graph, describing a problem, onto a few partial graphs, that can be maintained by different agents. Moreover, the multiagent's cooperation enables the application to the local graphs the graph transformation rules introduced for the centralizes graph; this permits us transfer all theoretical achievements of the centralized graph trans-formations to the distributed environment. The usefulness of the hierarchical graphs structure are and some examples of its usefulness are presented.
\end{abstract}

\section{Introduction}

Graphs are very useful formalism describing in a natural way a wide spectrum of problems. Unfortunately their use is limited with respect size and distribution. All the propositions mentioned in the three volume Handbook on Graph Grammars and Computing by Graph Transformations [1][2][3] remember the transformed graphs in one place. Moreover, the size of these graphs is limited due to a computational complexity of the parsers and the membership checkers. Even in the case of a few solutions, which offer the polynomial complexity of the solution of the mentioned problems (like $\mathrm{O}\left(\mathrm{n}^{2}\right)$ in [4]), this complexity permits us to think rather about hundreds or thousands nodes than about billions of ones. Let's note that billion of nodes is size of the small semantic web solutions.

The natural solution of the mentioned problem seems to be a graph distribution and a parallel transformation of these subgraphs. While the Multiagent Systems are characterized [5] by the assumption that:

- each agent has incomplete information or capabilities for solving the problem and, thus, a limited point of view,

- there is no global system control,

- data are decentralized,

- computations are asynchronous,

they seam to be the natural candidate for supporting distributed graph transformations. 
The first approach, of the author, at solving the similar problem [6] also assumes creation a set of agents; each of them maintains a local graph, and transforms it (what sometimes needs an cooperation with another agent). The presented solution of the distributed control of the allocation state assumes that the rules of the agents cooperation are designed for the given set of graph grammar production (describing graphs transformations); any change of this grammar causes the redesigning of the agents cooperation rules.

The GRADIS agent's framework, presented in the paper, enables the cooperation of the agents (maintaining a local graph) in a way, that is independent from the graph grammar definition. What is more, the cooperation of the local graph transformations systems, that are founded upon different types of graph grammars, is also possible.

The scope of the paper is the following: in section 2 the basic model of the Multiagent GRADIS framework is introduced; in section 3 the theoretical foundation of the complementary graph construction (supporting the graph distribution) is presented; in section 4 the way of the cooperation of agents using different types of grammars is considered; finally, some concluding remarks are presented.

\section{GRADIS Agent Model}

The GRADIS framework (that is an acronym of GRAph DIStribution toolkit) makes possible the distribution of a centralized graph and the controlling its behavior with the help of concurrent processes. The proposed solution is based on Multiagent technology; an agent is responsible both for:

- a modification of the maintained (local) graph, in a way described by the graph transformation rules associated with it.

- a cooperation with other agents for the purpose of holding the cohesion of the whole graph system.

The GRADIS agent model assumes the existence of two types of agents, called: maintainers and workers.

The maintainer agent - maintains the local graph; the whole set of maintainers take care about the global system cohesion understood as a behavior equivalent to the graph transformations made over the centralized graph. Initially we assume, that at the beginning one maintainer controls the centralized graph, but it is able to split itself onto the set of maintainer agents controlling parts of the previous graph transformation. The cooperation of the maintainers is based on the exchanging information among the elements of the agent's local graph structure; the graph transformation rules are inherited from the centralized solution. The formal background of the maintainer's activities is presented in section 3 .

The worker agents - are created: temporarily - for the purpose of realization of the given action (eg. for finding subpattern) or permanently - to achieve the more complex effect (eg. for detail designing of the element represented a the lower graph hierarchy). The worker graph structure is generated while its creation (by a maintainer or other worker agent) and is associated with some part of the parent's graph structure. However, this association in not the direct association among some nodes of maintained by these agents graph structure; we assume, that the parent worker 
association is made on the graph transformation level i.e. some worker's transformation enforces the realization of some graph transformation over the parent's graph structure. This problem will be in detail discussed in section 4 .

The GRADIS agent's model supports the hierarchical model structure with the following limitation:

1. a maintainer agent can exist only at the first level of this structure,

2. a maintainer cannot split itself when it is the parent of some workers,

3. a worker cannot split itself,

4. workers cooperation is limited only to worker parent relations.

The 1, 3, 4 assumptions seam to be the fundamental one, and we have not the intention to modify them in the next time. We would like to cancel the second assumption, and the algorithm of the assurance correct cooperation of the worker with the maintainer agents, created by splitting worker's parent is in the final phase of the realization.

\section{Complementary Graph as a Background for Maintainer Agent Work}

The data structure, that is maintained and transformed by agents, has a form of labeled (attributed) graphs. Let $\Sigma^{\mathrm{v}}$ and $\Sigma^{\mathrm{e}}$ be a sets; the elements of $\Sigma^{\mathrm{v}}$ are used as node labels and the elements of $\Sigma^{\mathrm{e}}$ are used as edge labels. The graph structure are defined as follows:

\section{Definition 3.1}

A $\left(\Sigma^{\mathrm{v}}, \Sigma^{\mathrm{e}}\right)$-graph is a 3-tuple $(\mathrm{V}, \mathrm{D}, \mathrm{v}$-lab) where $\mathrm{V}$ is nonempty set, $\mathrm{D}$ is a subset of $\mathrm{V} \times \Sigma^{\mathrm{e}} \times \mathrm{V}$, and $\mathrm{v}$-lab is a function from $\mathrm{V}$ into $\Sigma^{\mathrm{V}}$.

For any $\left(\Sigma^{\mathrm{v}}, \Sigma^{\mathrm{e}}\right)$-graph $\mathrm{G}, \mathrm{V}$ is set of nodes, D is set of edges and v-lab is a node labeling function. One can extend this graph definition eg. by introduction attributing functions both for nodes and edges, but these extensions will not influence on the rules of the centralized graph distribution and their transformation, because of that they will not consider here.

Our intention is splitting of the graph $G$ onto a few parts and distribute them onto different locations. Transformation of each subgraph $\mathrm{G}_{\mathrm{i}}$ will be controlled by some maintainer agent.

To maintain the compatibility of centralized graph with the set of split subgraphs some nodes (called border nodes) should be replicated and placed in the proper subgraph. Graphically, we will mark a border node by a double circle representation; we also introduce the set $\operatorname{Border}(\mathrm{G})$ to express that $\mathrm{v}$ is a border node in the graph $\mathrm{G}$ by a formulae $v \in \operatorname{Border}(G)$. During the splitting of the graph we are interested in checking if the connection between two nodes crosses a border among the subgraphs; the function PathS $(\mathrm{G}, \mathrm{v}, \mathrm{w})$ will return all sets of the nodes belonging to the edges creating a connection (without cycles) among v and w. For example for the graph $G$ presented in figure 3.1 PathS $(\mathrm{G}, \mathrm{a}, \mathrm{c})=\{\{\mathrm{a}, \mathrm{c}\},\{\mathrm{a}, \mathrm{b}, \mathrm{c}\},\{\mathrm{a}, \mathrm{d}, \mathrm{e}, \mathrm{c}\},\{\mathrm{a}, \mathrm{d}, \mathrm{f}, \mathrm{g}, \mathrm{e}, \mathrm{c}\}\}$. 


\section{Definition 3.2}

The set of graphs $G_{i}=\left(V_{i}, D_{i}, v-l a b_{i}\right)$, for $i=1 . . k$, is a split of graph $G$ to a complementary forms iff exist a set of injective homomorphisms $s_{i}$ from $G_{i}$ to $G$ such as :

1) $\bigcup_{\mathrm{i}=1 . \mathrm{k}} \mathrm{s}_{\mathrm{i}}\left(\mathrm{G}_{\mathrm{i}}\right)=\mathrm{G}$

2) $\forall \mathrm{i}, \mathrm{j}=1 . . \mathrm{K} \quad\left(\mathrm{s}_{\mathrm{i}}\left(\mathrm{V}_{\mathrm{i}}\right) \cap \mathrm{s}_{\mathrm{j}}\left(\mathrm{V}_{\mathrm{j}}\right)\right)=\left(\mathrm{s}_{\mathrm{i}}\left(\operatorname{Border}\left(\mathrm{G}_{\mathrm{i}}\right)\right) \cap \mathrm{s}_{\mathrm{j}}\left(\operatorname{Border}\left(\mathrm{G}_{\mathrm{j}}\right)\right)\right)$

3) $\forall \mathrm{w} \in \mathrm{V}_{\mathrm{i}} \quad \forall \mathrm{v} \in \mathrm{V}_{\mathrm{j}}: \quad \exists \mathrm{p} \in \operatorname{PathS}(\mathrm{G}, \mathrm{w}, \mathrm{v}) \Rightarrow \exists \mathrm{b} \in \operatorname{Border}\left(\mathrm{G}_{\mathrm{i}}\right): \mathrm{s}_{\mathrm{i}}(\mathrm{b}) \in \mathrm{p}$

4) $\forall \mathrm{j}=1 . . \mathrm{k} \quad \mathrm{v} \in \operatorname{Border}\left(\mathrm{G}_{\mathrm{j}}\right) \Leftrightarrow\left(\exists \mathrm{w} \in \mathrm{G}_{\mathrm{j}}: \mathrm{w} \leftrightarrow \mathrm{v}\right)$ or $\mathrm{G}_{\mathrm{j}}=\{\mathrm{v}\}$

where $\leftrightarrow$ means that the nodes are connected by an edge.

The introduced formal definition is difficult to use in practical construction of the complementary graphs, because of that we introduce an algorithm for splitting of the centralized graph.

\section{Algorithm 3.1}

Let $H$ be a subgraph of $G$ then two complementary graph $H^{\prime}$ and $H$ ', are created in the following steeps:

1. initially $\mathrm{H}^{\prime}=\mathrm{H}$ and $\mathrm{H}^{\prime}=\mathrm{G} \backslash \mathrm{H}$

2. for every $v \in H$ such that exist $w \in G \backslash H$ if $w$ is connected with $v$ then $v$ is replicated and:

- v stays in H' but it is marked as a border node,

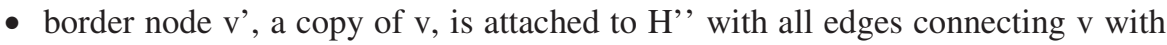
G\H,

- proper references are added to $\mathrm{v}$ and v' (iff $\mathrm{v}$ has been a border node before this operation this references should also associate these node with another ones),

3. some reindexation of $\mathrm{V}_{\mathrm{H}}$, and $\mathrm{V}_{\mathrm{H}}$, should be made for optimizing local transformations.

The unique addressing of nodes in the glued graph (after 3-th steep of an algorithm or any sequences of local graph transformations) is guaranteed by the remembering their indices as a pair (local_graph_id, local_index). We also assume, that marking of the node as a border one is associated with designation for it of an unique index in the border nodes set (border_id is equal to 0 ).

\section{Algorithm 3.2}

The construction of $G$ from the complementary graphs set $\left\{G_{i}\right\}$ is made in the following way:

- for the boarder nodes, one of the replicas, indexed as (0,glob_bord_node_index), is added to $\mathrm{V}$,

- foe all normal (not border) nodes are added to $\mathrm{V}$, with their local indexation,

- the edges in $\mathrm{E}$ are inherited from the local graphs (if one node of the edge is a border node in the final edge its global representative appears).

- The labeling function lab is the union of $\operatorname{lab}_{\mathrm{i}}$.

Splitting the graph $\mathrm{G}$ onto a few new complementary forms can be made by execution of the algorithm 3.1 on the already split graph H' or H' (and so on). 
An example of $\mathrm{G}$ and set of two comple-mentary distributed graphs are presented appropriately on Fig. 3.1 and 3.2. The indexation inside $G$ is consistent with one introduced in algorithm 3.2.

For any border node $\mathrm{v}$ in the graph $\mathrm{G}_{\mathrm{i}}$ we can move boundary in such a way that, all nodes that are connected with $\mathrm{v}$ (inside another complementary graphs) are incorporated to $\mathrm{G}_{\mathrm{i}}$ as a border nodes and the $\mathrm{v}$ node replicas are removed from another graphs (i.e. $\mathrm{v}$ stays a normal node). For graphs presented in Fig. 3.2 an incorporate $((0,1), 1)$ operation creates graphs presented in Fig. 3.3.

Let $\mathbb{G}$ be a class of graphs, then analogically as in [7], we define $\mathbb{R}$ as a class of rules and $\Rightarrow_{\mathrm{r}}$ as a rule application operator, that for $r \in \mathbb{G} \times \mathbb{G}$ yielding a binary operation over the graphs. A pair $\left(\mathrm{G}, \mathrm{G}^{\prime}\right)$, usually written as $\mathrm{G} \Rightarrow \mathrm{r}_{\mathrm{r}}$, establish a direct derivation from $G$ to $G$ ' through $r$. The GRADIS framework associates with each distributed complementary graph the maintainer agent, that not only makes the local derivations possible, but also assures the coordination and synchronization of parallel derivations on the different

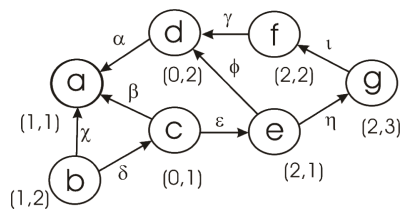

Fig. 3.1 Graph G
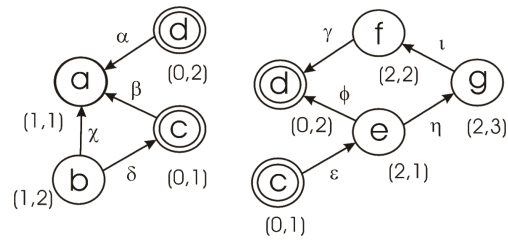

Fig 3.2 Complementary graphs

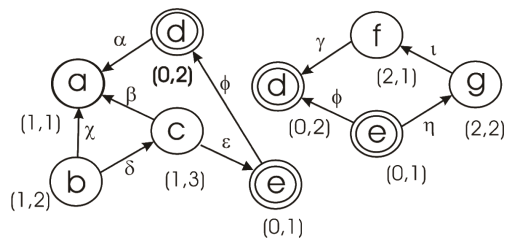

Fig. 3.3. Complementary graphs- 2 complementary graphs. Each maintainer agent is also able to gather (after some cooperation with other agents) a temporary information about the neighborhood of the replicas of the pointed boundary node; for any natural number $\mathrm{k}$ and any border node $\mathrm{v} \in \operatorname{Border}\left(\mathrm{G}_{\mathrm{i}}\right)$ i-th maintainer agent is able to return a graph $\mathrm{B}=\mathrm{k}$-distance neighborhood(v) that is a subgraph of the global graph $\mathrm{G}$ limited to the nodes distant from $\mathrm{v}$ no more then $\mathrm{k}$.

Graph grammars provide a mechanism in which a local transformations on a graph can be modeled in a mathematically precise way. The main component of a graph grammars is a finite set of productions; a production is in general, a triple $(\mathrm{L}, \mathrm{R}, \mathrm{E})$ where L and R (left- and right-hand graph of production, respectively) are a graph and $\mathrm{E}$ is some embedding mechanism. Such a production can be applied to graph $\mathrm{G}$ whenever there is an $m$ occurrence of $L$ in $G$. It is applied by removing $m(L)$ from $G$, replacing it by (an isomorphic copy) of $\mathrm{R}$, and finally using embedding mechanism $\mathrm{E}$ to attach $\mathrm{R}$ to the remainder $\mathrm{G}-\mathrm{m}(\mathrm{L})$.

The general idea of the proposed application of the production $\mathrm{L} \rightarrow(\mathrm{R}, \mathrm{E})$ over a distributed graph is the following: 
1. each of i-th maintainer agent autonomously decide to apply this production when one can find the $m$ occurrence of $L$ inside $G_{i}$, none of border nodes are removed and all removed edges belongs to $\mathrm{G}_{\mathrm{i}}$ edges.

2. otherwise, i-th maintained agent needs the cooperation with the rest of the system, that will be made in three steep:

2.1. the gathering an information: for all nodes $v$ such that $\mathrm{v} \in \operatorname{Border}\left(\mathrm{G}_{\mathrm{i}}\right) \cap \operatorname{Nodes}(\mathrm{m}(\mathrm{L}))$ we are looking such $\mathrm{k}$ that the graph B designnated as k-distance_ neighborhood(v) covers L and all removed nodes and edges.

2.2. the preparing an environment: all nodes belongs to $B$ and does not belongs to $\mathrm{G}_{\mathrm{i}}$ are incorporated to the graph $\mathrm{G}_{\mathrm{i}}$ by execution of sequence incorporate $(\ldots)$ operations made in the transactional mode.

2.3. the applying of a production: a production $\mathrm{L} \rightarrow(\mathrm{R}, \mathrm{E})$ can be applied in a new created graph $\mathrm{G}_{\mathrm{i}}$ ' according to 1-th rule (local derivation).

The presented algorithm does not depend on the specific properties of the graph transformation mechanism like NLC embedding transformation (in case of the algorithmic approach) [8] or single- and double-pushout (in the case of the algebraic approach) [9]. In [10] the cooperation among the agents in the case of these types graph transformations is considered and a detail algorithms basing on these transformation properties are presented.

\section{Cohesion Graph Grammar as a Background for Workers Agents Cooperation}

The maintainer cooperation, based on complementary graphs, make possible to distribute some centralized solution onto the distributed agent's environment; each of agents maintains the "reasonable" part of the centralized graph, what makes the parallel computation effective. As it was mentioned in section 2, GRADIS framework offers more one type of agents - the worker agents. The worker agent is created by the maintainer agent temporarily or permanently to support the maintainer creativity. For a better understanding of the problem we consider some examples.

The first example is a part of the problem of finding subpatterns, presented in [11]. Let, set of maintainer agents remember a set of complementary graphs $\left\{G_{i}\right\}$ describing large pattern, and let the graph grammar SUB defines a set subpatterns; we would like to check iff a given node $\mathrm{v}$ of $\mathrm{G}_{\mathrm{i}}$ graph is a starting point of any subpattern (defined by SUB) contained by $\mathrm{H}=\bigcup \int \mathrm{G}_{\mathrm{i}}$.

For the simplicity we assume that in all subpatterns minimal distance between two node is less or equal to $\mathrm{k}$. Usually, graph $\mathrm{B}=\mathrm{k}$-distance_neighborhood(v) in $\mathrm{H}$ does not contain itself in the graph $\mathrm{G}_{\mathrm{i}}$. The sequence of incorporate operations can enlarge graph $G_{i}$ to $G_{i}$ ' such that $B \subseteq G_{i}$ ', but this solution seems unlucky from two reasons:

- it causes growing the i-th maintainer structure and finally it leads to the centralized solution, 
- the i-th maintainer agent should in parallel way service two types graph grammars transformations (inherited from centralized solution and defined by SUB graph grammar) what would enforce the need of these activities synchronization. This synchronization could not be independent from the definition of the mentioned grammars.

- It seams to be better replicate the structure of graph B, and create a worker agent that solves the membership problem parameterized by graph B, node $\mathrm{v}$, and graph grammar SUB ${ }^{1 .}$ Let's consider maintainer-worker relationship:

- the worker is created only for realization of the particular task,

- the maintainer and worker structures are not synchronized,

- the worker after the task realization informs the maintainer about its work effects.

In GRADIS we assume that the communication among these agents is made at the graph transformation level, i.e. the worker recommends execution by the maintainer of some transformations depending on the final effect of its work.

The second example is inspired by a distributed adaptive design process [12]. This process composes from a few phases: firstly we create basic plans (eg. of a building design), next we can plan rooms arrangement and finally details of the furniture. Graph transformations seams to be very promising for this application [13].

We assume that, the building plain (in a graph form) is maintained for the set of maintainer agents, and each of maintainer agents takes care about the subgraph responsible for parameterization (allocation of walls, doors, windows, etc.) of one or more rooms. With each of the rooms it is associated a permanent worker agent that is responsible for this room arrangement. Let's note that, in this case, the maintainer-worker relationship is more complex then in the previous example. The moving walls or doors has a very strong influence on the room arrangement process and from the other hand putting of the wardrobe on the wall with door is possible only when these door will become transferred into other place. Thus the cooperation between graph transformation systems supported by maintainer and worker agents should be very close.

The formal background of the for the support above problem bases on a conjugated graph grammars theory [14][15]. In the conjugated graph a new type of nodes appears - remote nodes. Remote nodes represent the nodes appearing in other graph structures. In the conjugated graphs grammars we assume that, the $\mathrm{P}$ graph transformations (on the first agent graph structure) in which exist a remote node $\mathrm{w}$ is associated with the Q graph transformation (on the second agent graph structure), such that it modifies the neighborhood of the node represented by $\mathrm{w}$. The pair P and Q are called conjugated transformations in context of remote node w. In order to synchronize the set of conjugated graph transformations we assume that, GRADIS assures that both $\mathrm{P}$ and $\mathrm{Q}$ graph transformations will be successfully performed.

To guarantee this we consider three types of the conjugated graph grammars:

- the strictly conjugated graph grammars - when the created conjugated graph structure guarantee, that application of $\mathrm{P}$ graph transformation forces the possibility of application Q graph transformation. In [15] there is proved that the fulfillment eight condition guarantee strictly conjugated model for double-pushout graph grammars.

${ }^{1}$ It is assumed that SUB graph grammar is one of that are able to solve the membership problem in a polynomial time complexity (like for example ETPL(k) graph grammar [16]). 
- the conditional conjugated graph grammar - when both the transformations are performed, when some (earlier defined) conditions are fulfilled by the both graph structures.

- the transactional conjugated graph grammars - when both the production are performed in the transactional mode i.e. either all of these graph transformation can be performed without violating the conjugated graph cohesion or none of them will be applied.

Let's notice that in conditional and transactional models, there was not any assumption on the type graph transformation performed by agents. There is no objection to construct a system in which parent and son agents uses different graph transformation systems.

The level of a furniture design (supported by the worker created for the support the room arrangement) points out yet another problem. Assume that, we would like to furnish the house in the style Louise XVI and all chairs should be identical. The first part of the problem can be solved by preparing of the proper graph grammar. The second at the first sight demands the non-hierarchical worker's cooperation. Fortunately, it can be solved by introduction worker's equivalence, i.e. while a worker creation its parent can decide whether create a new agents instance or to use already created agent. The common agent react on demand any of its parents, but its reaction is broadcasted to all of them. This solves the problem of some subsystems unification; in our example when the one room arranger suggest made chairs more wide, then this modification (if accepted by the other arrangers) will be made for all chairs.

\section{Conclusions}

There was a few fundamental assumptions of the agents GRADIS framework:

- the introduction of the possibility graph transformation over the set of distributed graphs,

- the making use of the all earlier theoretical achievement of the centralized graph transformations in the multiagent's environment.

- the support the agents cooperation over the hierarchical graph structures.

The concept of the distribution (to specify a concurrent and distributed system) was initially considered in the algebraic approach. The distributed graph transformation [2] was developed with the aim to naturally express computation in system made of interacting parts. In general, it is assumed an existence of some global state, representted as a graph, that is used to synchronize execution of the amalgamated productions or synchronized productions. In [9], a distributed attributed graph (Distr(AGr) has two levels: a network and a local level. Network nodes are assigned to the local graphs, which represent their state. This graphs are transformed via distributed rules. They consist from a network rule and a set of local rules, one for each node of network rule. In addition to the usual, in the double push-out approach, dangling and identification conditions, two additional locality conditions should be verified [17][18]. The satisfying of the locality conditions by a local graph morphism assures that the pushout can be constructed component wise. There are a few examples of usefulness of 
Distr(AGr) in the visual design of distributed system [17], the specifying integrated refactoring [19], the modeling a analysis of a distributed simulation protocols [20]. The problem of evaluation of the distributed rules and graphs in a real distributed environment is not up till now considered (the last implementation [20] is made in a centralized environment with help of AToM tool [21]).

The complementary graphs model, introduced for the worker agents in the GRADIS framework, assure that for each of the local graphs any graphs transformation are introduced locally using only these graph; what means that GRADIS does not influence of the properties of the graph transformation (considered in context of global graph generation) such as: confluention, Local Church-Roster problem; the explicit parallelism problem is also solved on condition, that concurrency instead real parallelism is possible, because we assume that, the evaluation of $m\left(L_{i}\right)$ is made in a critical section with respect of the nodes belonging to $\mathrm{m}\left(\mathrm{L}_{\mathrm{i}}\right)$.

The conjugate graph grammars system, supporting the hierarchical graph transformation systems, assumes that each agent can be supported by another type of graph transformation. As a consequence, for each of the subproblem we can use the graph transformation system that describes it in the best way.

The different graph transformation systems, coexisting in a hierarchical graph structure, seams to be promising solution with respect the final computational effectiveness evaluation; it is obvious that the graph structure supported by the maintainer agents, with respect of its size, should be managed by graph transformation systems with the polynomial time complexity. Unfortunately, such systems have too weak descriptive power to describe most of the considered problems. On the other hand, the maintainer usually keep an information about the structural properties of the modeled system; for such type of information exist at least one class of graph transformation systems (ETPL(k) graph grammars [16]) with enough descriptive power [22] and the polynomial time complexity of the parsing and membership checking. From the worker agents we need not pay such attention on the graph transformation complexity, when the size of supported graphs is limited to tens. Finally, the computational complexity of such a hierarchical system, constructed in such a way, can be acceptable.

One of the most important limitation of the current GRADIS framework is lack possibility of the maintainer's graph split, when some worker has been created by them (then more that some of this worker could be created as a permanent one). This problem is our preferential work, and an algorithm of the assurance correct cooperation of the worker with maintainer agents, created by splitting the worker's parent seams to in the final phase of the realization.

\section{Reference}

1. Rozenberg, G.: Handbook of Graph Grammars and Computing By Graph Trans-formation, Foundations edn., vol. I. World Scientific Publishing Co., Singapore (1997)

2. Ehrig, H., Engels, G., Kreowski, H.-J., Rozenberg, G.: Handbook of Graph Grammars and Computing By Graph Transformation, Application, Languages and Tools edn., vol. II. World Scientific Publishing Co, Singapore (1999)

3. Ehrig, H., Kreowski, H.-J., Montanari, U., Rozenberg, G.: Handbook of Graph Grammars and Computing By Graph Transformation, Concurrency, Parallelism, and Distribution edn., vol. III. World Scientific Publishing Co, Singapore (1999) 
4. Flasiński, M.: Distorted Pattern Analysis with the Help of Node Label Controlled Graph Languages. Pattern Recognition 23(7), 765-774 (1990)

5. Sycara, K.P.: Multiagent Systems. AI Magazine, 79-92 (1998)

6. Kotulski, L.: Supporting Software Agents by the Graph Transformation Systems. In: Alexandrov, V.N., van Albada, G.D., Sloot, P.M.A., Dongarra, J. (eds.) ICCS 2006. LNCS, vol. 3993, pp. 887-890. Springer, Heidelberg (2006)

7. Kreowski, H.-J., Kuske, S.: Graph Transformation Units and Module. In: [2], pp. 607-640

8. Engelfriet, J., Rozenberg, G.: Node Replacement Graph Grammars. In: [1], 3-94

9. Ehrig, H., Heckel, R. Löwe, M., Ribeiro, L., Wagner, A.: Algebraic Approaches to Graph Transformation - Part II: Single Pushout and Comparison with Double Pushout Approach. In: [1], pp. 247-312

10. Kotulski, L.: On the Distribution Graph Transformations, Preprint of Automatics Chair, AGH University of Science and Technology (January 2007); (submitted to the Theoretical Computer Science)

11. Kotulski, L.: Distributed Graphs Transformed by Multiagent System. In: International Conference on Artificial Intelligence and Soft Computing ICAISC, Zakopane. LNCS(LNAI), vol. 5097. Springer, Heidelberg (2008) (accepted) (to be published)

12. Strug, B., Kotulski, L.: Distributed Adaptive Design with Hierarchical Autonomous Graph Transformation Systems. In: Shi, Y., van Albada, G.D., Dongarra, J., Sloot, P.M.A. (eds.) ICCS 2007. LNCS, vol. 4488, pp. 880-887. Springer, Heidelberg (2007)

13. Grabska, E., Strug, B.: Applying Cooperating Distributed Graph Grammars in Computer Aided Design. In: Wyrzykowski, R., Dongarra, J., Meyer, N., Waśniewski, J. (eds.) PPAM 2005. LNCS, vol. 3911, pp. 567-574. Springer, Heidelberg (2006)

14. Kotulski, L., Fryz, Ł.: Assurance of system cohesion during independent creation of UML Diagrams. In: Proceedings at the Second International Conference on Dependability of Computer Systems DepCoS - RELCOMEX 2007, Poland, June 14-16, 2007, pp. 51-58. IEEE Computer Society, Los Alamitos (2007)

15. Kotulski, L., Fryz Ł.: Conjugated Graph Grammars as a Mean to Assure Consistency of the System of Conjugated Graphs. In: Third International Conference on Dependability of Computer Systems DepCoS - RELCOMEX 2008 (accepted, 2008)

16. Flasinski, M.: Power Properties of NLC Graph Grammars with a Polynomial Mem-bership Problem. Theoretical Comp. Sci. 201(1-2), 189-231 (1998)

17. Fisher, I., Koch, M., Taentzer, G., Vohle, V.: Distributed Graph Transformation with Application to Visual Design of Distributed Systems. In: [3], pp. 269-337

18. Taentzer, G.: Distributed Graphs and Graph Transformation, Applied Categorical Structures. Special Issue on Graph Transformation 7(4) (December 1999)

19. Bottoni, P., Parisi Presicee, F., Taentzer, G.: Specifying Integrated Refactoring with Distributed Graph Transformations. In: Pfaltz, J.L., Nagl, M., Böhlen, B. (eds.) AGTIVE 2003. LNCS, vol. 3062, pp. 220-235. Springer, Heidelberg (2004)

20. de Lara, J., Taentzer, G.: Modelling and Analysis of Distributed Simulation Protocols with Distributed Graph Transformation. In: Proceedings of the Fifth international Conference on Application of Concurrency To System Design. ACSD, pp. 144-153. IEEE Computer Society, Washington (2005)

21. de Lara, J., Vangheluve, H.: AToM3: A Tool for Multi-Formalism Modeling and MetaModelling. In: Kutsche, R.-D., Weber, H. (eds.) FASE 2002. LNCS, vol. 2306, pp. 174 188. Springer, Heidelberg (2002)

22. Kotulski, L.: Graph representation of the nested software structure. In: Sunderam, V.S., van Albada, G.D., Sloot, P.M.A., Dongarra, J. (eds.) ICCS 2005. LNCS, vol. 3516, pp. 1008-1011. Springer, Heidelberg (2005) 